\title{
Estudio de Perfiles Motivacionales Latentes Asociados con la Satisfacción y Autoeficacia Académica de Estudiantes Universitarios
}

\author{
Study of Latent Motivational Profiles Associated with Academic Satisfaction and \\ Academic Self-Efficacy of University Students
}

\author{
Milenko Del Valle $^{1}$, Jorge Vergara ${ }^{2}$, Ana B. Bernardo $^{3}$, Alejandro Díaz ${ }^{4}$ y Inés Gahona Herrera ${ }^{5}$
}

\begin{abstract}
Resumen
Los estudiantes que desarrollan altos grados de autodeterminación para lograr los aprendizajes tienen una percepción positiva de su autoeficacia académica y altos niveles de satisfacción con la experiencia académica. Por lo tanto, es necesario establecer qué variables tienen relación directa con la conformación de perfiles motivacionales que afectan los procesos de aprendizaje. El objetivo del estudio fue identificar los perfiles motivacionales latentes de estudiantes universitarios, evaluando su relación con la satisfacción y la autoeficacia académica. Los participantes fueron 437 estudiantes universitarios. La identificación de subgrupos se realizó utilizando el análisis de perfil latente. Los resultados apoyaron el ajuste de un modelo de cuatro perfiles motivacionales: alta cantidad $(n=84)$, baja calidad $(n=28)$, baja cantidad $(n=35)$, y alta calidad ( $\mathrm{n}=290$ ). Estos hallazgos confirman que los grupos con mayores grados de autodeterminación para el aprendizaje muestran los niveles más altos de satisfacción y autoeficacia académica.
\end{abstract}

Palabras clave: motivación, satisfacción, autoeficacia, estudiantes universitarios, análisis de perfil latente

\begin{abstract}
Students who develop high degrees of self-determination to achieve learning, have a positive perception of their academic self-efficacy and high levels of satisfaction with academic experience. Therefore, it is necessary to establish which variables are directly related to the conformation of motivational profiles that affect learning processes. The aim of the study was to identify the latent motivational profiles of university students, assessing the relationships with satisfaction and academic self-efficacy. The participants were 437 university students. Subgroups identification was performed using latent profile analysis. The results supported the emergence of four motivational profiles: high quantity $(n=84)$, low quality $(n=28)$, low quantity $(n=35)$, and high quality $(n=290)$. These findings confirm that the groups with the highest degrees of self-determination for learning show the highest levels of academic satisfaction and academic self-efficacy.
\end{abstract}

Keywords: motivation; satisfaction, self-efficacy, university students, latent profile analysis

Este trabajo es parte del proyecto FONDECYT No 1161502 titulado "Modelo explicativo de la permanencia y el abandono de los estudios universitarios, basado en procesos cognitivos-motivacionales".

\footnotetext{
${ }^{1}$ Doctor en Psicología, Universidad de Antofagasta, Profesor Asociado de la Facultad de Ciencias Sociales, Artes y Humanidades, Avda. Universidad de Antofagasta s/n, Chile. Tel.: +56(55) 2637249. Correo: mdelvalle@ uantof.cl, https://orcid.org/0000-0003-3739-1001

${ }^{2}$ Doctor en Psicología, Académico disciplinar, Facultad de Ciencias Sociales, Universidad de Las Américas, sede Concepción, Av. Chacabuco 539, Chile. Tel.: +56(41)2129968. Correo: jvergaram@udla.cl, https://orcid.org/0000-0003-3655-813X

${ }^{3}$ Doctora en Psicología, Profesora Titular del Departamento de Psicología, Universidad de Oviedo, Plaza Feijoo s/n, España. Tel.: 985109541. Correo: bernardoana@uniovi.es, https://orcid.org/0000-0001-5984-0985

${ }^{4}$ Doctor en Psicología, Universidad de Concepción, Profesor Titular de la Facultad de Ciencias Sociales, Victor Lamas 1290, Chile. Tel.: +56(41) 2204301. Correo: adiazm@udec.cl, http://orcid.org/0000-0002-3090-5463

${ }^{5}$ Licenciada en Psicopedagogía, Académica de la Universidad de Antofagasta, Facultad de Ciencias Sociales, Artes y Humanidades, Avda. Universidad de Antofagasta s/n, Chiel. Tel.: +56(55) 2637249. Correo: ines.gahona@uantof.cl, https://orcid.org/0000-0003-2431-1201

Revista Iberoamericana de Diagnóstico y Evaluación - e Avaliação Psicológica. RIDEP · N57 · Vol.4 · 137-147 · 2020

ISSN: 1135-3848 print /2183-6051online
} 


\section{Introducción}

Las investigaciones sobre los factores que intervienen en el proceso de aprendizaje de los estudiantes destacan la importancia que adquieren las variables cognitivo-motivacionales como mediadoras del éxito o del fracaso académico (Miñano \& Castejón, 2011).

Distintos estudios reconocen la relevancia que adquiere la motivación académica, que hace referencia a aquellos factores internos que energizan, impulsan y direccionan al estudiante a realizar una serie de actividades propuestas en el proceso de aprendizaje, actuando como mediadoras en la adquisición de los contenidos curriculares, persistencia y el desempeño académico (Stover et al., 2014; Valenzuela et al., 2015).

Se reconoce que la etapa de transición entre la educación secundaria y la universitaria es uno de los hitos en la vida del estudiante, por lo que es necesario atender al carácter multifactorial de este proceso, ya que pueden generar estados de incertidumbre y malestar, afectando su desempeño académico y la posibilidad de permanecer o abandonar los estudios universitarios (Álvarez \& López, 2017).

En Chile esta situación ha despertado un interés generalizado, debido al dinamismo que está experimentado la formación universitaria, sobre todo, si se considera que en promedio la mitad de los estudiantes que ingresan a la educación superior abandonan sus estudios antes de llegar a finalizar su formación profesional, presentándose con mayor fuerza en los primeros años de estudios (Donoso \& Schiefelbein, 2007; SIES, 2014).

Al respecto, resulta importante integrar componentes cognitivos y motivacionales para conocer el complejo proceso del aprendizaje, las dificultades que se presentan y los resultados que se obtienen, más aún, si se asume el aprendizaje como un proceso activo, donde los estudiantes deben reconocer y regular sus cogniciones y motivaciones (Alonso-Tapia, Panadero, \& Ruiz 2014; Rosário, 2012).

Debido a lo anterior, es necesario contribuir con evidencias que permitan establecer los distintos perfiles motivacionales de los estudiantes y la relación de la satisfacción académica, la satisfacción de las necesidades psicológicas básicas y la autoeficacia académica como variables que influyen en la motivación y el desempeño académico universitario.

Desde la perspectiva cognitiva, la teoría de las metas de logro entiende la motivación como representaciones mentales que realizan los estudiantes sobre la interacción entre el contexto y sus objetivos de desarrollo personal (Salmerón, Gutiérrez, Salmeron, \& Rodríguez, 2011). Esta perspectiva relaciona distintos tipos de metas para explicar la influencia en la motivación y el desempeño académico en diferentes áreas curriculares (Méndez-Giménez, Fernández-Río, \& Cecchini-Estrada, 2012; Valle et al., 2008).

Las investigaciones muestran la relación que existe entre las metas de estudio y los niveles de implicación académica, uso de estrategias de aprendizaje, patrones motivacionales, así como la utilización de estrategias adaptativas y niveles de implicación cognitiva, lo que demuestra que los estudiantes que poseen metas de aprendizaje aprenden más que aquellos que se orientan solo al desempeño (Meece, Anderman E., \& Anderman, L., 2006; Pérez-Villalobos et al., 2009; Valenzuela et al., 2015).

No obstante, aún falta por establecer cómo se logra que estudiantes motivados por el desempeño logren un tipo de motivación autodeterminada hacia el aprendizaje.

Existe evidencia de la importancia que tiene la motivación académica en el proceso de aprendizaje, en el desempeño y la satisfacción académica de los estudiantes, no obstante, la manera de entender la motivación y su relación con los resultados de aprendizaje responde a distintas perspectivas y marcos teóricos (Moreno, Silveira, \& Alias, 2015). Por lo tanto, es necesario construir antecedentes específicos relacionados con los perfiles motivacionales y las variables que influyen y afectan el proceso de aprendizaje.

El estudio de la motivación en el ámbito académico es un tema de creciente interés en la actualidad, ya que las investigaciones plantean que estudiantes caracterizados por altos niveles de motivación hacia el estudio y por la utilización de estrategias motivacionales adaptativas, se implican más en el proceso de aprendizaje destinando un mayor esfuerzo, lo que propicia a su vez un nivel de rendimiento académico y de 
satisfacción superior (Anaya-Durand \& AnayaHuertas, 2010).

De hecho, las investigaciones indican que el bajo desempeño académico no se relaciona de forma exclusiva con el nivel intelectual del alumno, sino más bien con la motivación y otros aspectos relacionados con ésta, como por ejemplo, la evaluación, la Figura del docente, el nivel cognitivo personal, el contexto ambiental, etc. (Bernardo et al., 2018).

Un importante mediador cognitivo y que influye sobre la motivación, el aprendizaje y el desempeño académico es la autoeficacia académica, entendida como un componente motivacional referido a las propias creencias que tienen los estudiantes de la capacidad para aprender o rendir efectivamente (BecerraGonzález \& Reidl, 2015; Oriol, Mendoza, Covarrubias, \& Molina, 2017).

Específicamente, las creencias o percepciones de autoeficacia académica de los estudiantes se consideran predictores significativos del desempeño, logro o éxito académico (Cartagena, 2008; Maroño, Deus, Mallo, \& Méndez, 2015), alta relación con el bienestar psicológico, emociones positivas, apoyo a la autonomía y facilitador del proceso de aprendizaje (Silva et al., 2014; Oriol et al., 2017).

Se ha observado que las creencias de autoeficacia que poseen los estudiantes, en distintos niveles educativos, permite conFigurar distintos perfiles motivacionales, así como una relación significativa con la autoestima y la motivación autodeterminada, predictores tanto del aprendizaje como del desempeño académico (Komarraju \& Dial, 2014; Valle et al., 2015).

Una de las teorías motivacionales actuales es la teoría de la autodeterminación, que logra superar la orientación exclusiva hacia la meta o logro académico y que define la motivación como el resultado de las tendencias internas del sujeto que le impulsan a realizar conductas en ausencia de recompensas externas, esta propuesta se sustenta en la idea del ser humano como agente causal y orientado para el crecimiento, con una tendencia natural para integrar sus elementos psíquicos en un todo coherente (Ryan \& Deci, 2000).

Esta teoría reconoce que la motivación (autónoma y controlada) tiene una naturaleza multidimensional con distintos niveles situados en un continuo, que va desde los niveles más altos de autodeterminación (regulación intrínseca) hasta el nivel más bajo de motivación (regulación extrínseca), encontrándose entre ellas distintos tipos de regulación que orientan el comportamiento (Vansteenkiste et al., 2018).

Para el caso de la motivación autónoma, entendida como la propia energía impulsada por el individuo para desempeñar una actividad, está constituida por los tipos de regulación intrínseca e identificada. Para el caso de la motivación controlada, entendida como las razones externas que presionan el comportamiento del individuo, se identifican los tipos de regulación introyectada y externa (Howard, Gagné, \& Bureau, 2017; Litalien et al., 2017).

La teoría de la autodeterminación propone que la energía del comportamiento de los individuos se genera por la búsqueda de la satisfacción de las necesidades psicológicas básicas de autonomía, competencia y relación sin importar las diferencias individuales, contextuales y culturales, por lo que su satisfacción es indispensable para un buen funcionamiento y para un desarrollo psicológico óptimo (Deci \& Ryan, 2008; Chen et al., 2015).

La necesidad de autonomía hace referencia a cuando la persona siente que es la causante de su conducta, no significa independencia o libertad total, sino más bien aceptación interna y compromiso con la conducta motivada, la competencia se da cuando la persona se siente eficaz con su conducta, logra interactuar efectivamente con el entorno experimentando la oportunidad para expresar o desarrollar sus capacidades y emprende nuevas tareas que le suponen un desafío, finalmente la necesidad de relación se produce cuando una persona se siente vinculada o comprendida por los demás, están relacionadas con autenticidad con otros significativos y experimentan un sentido de pertenencia (Deci \& Ryan, 2000; Tian, Chen, \& Huebner, 2014).

Desde esta perspectiva teórica, un estudio realizado con estudiantes universitarios chilenos analizó la formación de distintos perfiles motivacionales considerando la percepción de apoyo a la autonomía y la motivación académica. Los resultados mostraron la formación de cuatro 
perfiles motivacionales: (a) mala calidad, (b) baja cantidad, (c) buena calidad y (d) alta cantidad. Además, los resultados indicaron que los estudiantes agrupados en los perfiles con mayores niveles de autonomía para el aprendizaje presentaron los niveles más altos de satisfacción académica (Vergara-Morales et al., 2019).

Sánchez-Oliva et al., (2015) realizaron un estudio con estudiantes secundarios españoles, donde la formación de perfiles motivacionales se efectuó incorporando las medidas de satisfacción y frustración de las necesidades psicológicas básicas, al igual que el estudio realizado con estudiantes chilenos, los resultados mostraron la existencia de cuatro perfiles motivacionales: (a) mala calidad, (b) baja cantidad, (c) alta cantidad y (d) buena calidad.

Estos hallazgos coinciden con diferentes trabajos que plantean la existencia de un perfil motivacional autodeterminado (buena calidad), caracterizado por niveles altos en las regulaciones autónomas y un perfil no autodeterminado (mala calidad), caracterizado por niveles bajos en las regulaciones autodeterminadas y altas puntuaciones en las regulaciones controladas.

Además, los estudios apoyan la existencia de un perfil denominado alta cantidad, caracterizado por altas puntuaciones en las regulaciones autodeterminados y no autodeterminados, y un perfil denominado baja cantidad, caracterizado por bajas puntuaciones en las regulaciones autodeterminados y no autodeterminados (Rothes, Lemos, \& Gonçalves, 2017; Wormington, Corpus, $\&$ Anderson, 2012).

Los antecedentes muestran que en el contexto universitario chileno no se dispone de antecedentes sobre la formación de perfiles motivacionales que incluyan la satisfacción académica y las creencias de autoeficacia académica. Debido a esto, el objetivo del estudio fue identificar los perfiles motivacionales de estudiantes universitarios, evaluando la relación con la satisfacción y la autoeficacia académica.

Con este trabajo se espera contribuir de manera específica con dos temas para nuestro medio; en primer lugar, se busca ampliar el conocimiento científico respecto a la importancia que tiene para el aprendizaje en el contexto universitario, la satisfacción académica y las creencias de autoeficacia.
En segundo lugar, se busca reconocer la conFiguración de distintos perfiles motivacionales latentes en estudiantes universitarios y la influencia que tiene en esta conFiguración motivacional la satisfacción académica y las creencias de autoeficacia académica.

\section{Método}

\section{Participantes del estudio}

Un total de 437 estudiantes universitarios de la Universidad de Antofagasta, con edades comprendidas entre los 18 y 31 años, con una edad media de 19.5 años ( $\mathrm{DE}=1.78)$. Un 61,3\% de los participantes fueron mujeres $(n=268)$ y un $38,7 \%$ fueron hombres $(n=169)$. El muestreo utilizado fue no probabilístico incidental. Los estudiantes participaron de manera voluntaria en el estudio y pertenecen a cuatro facultades: Ciencias de la salud, Ciencias del mar, Ingeniería y Medicina.

\section{Instrumentos de medición}

Se utilizaron tres escalas que evaluaron la motivación académica, la satisfacción académica y la autoeficacia académica.

Escala de autorregulación académica (Vansteenkiste et al., 2009). Se compone de 16 ítems que miden los motivos para involucrarse en las actividades académicas. La escala se constituye de cuatro factores: (a) regulación intrínseca (4 ítems, p. ej.: "Porque es divertido), (b) regulación identificada (4 ítems, p. ej.: "Porque quiero aprender cosas nuevas"), (c) regulación introyectada (4 ítems, p. ej.: "Porque me sentiría culpable si no estudiara"), y (d) regulación externa (4 ítems, p. ej.: "Porque se supone que debo hacerlo") (Vansteenkiste et al., 2009). Este instrumento ha mostrado niveles adecuados de validez y consistencia interna (Vansteenkiste et al., 2009; Vansteenkiste et al., 2012). Para el desarrollo del estudio se aplicó la versión en español utilizada por Vergara-Morales (2019). Se consideró la medida de motivación autónoma, calculada mediante el promedio de las puntuaciones de los sub-componentes regulación intrínseca y regulación identificada. Además, se consideró la medida de motivación controlada, calculada a través del promedio de las 
puntuaciones de los sub-componentes regulación introyectada y regulación externa. Los ítems se respondieron en una escala de 7 puntos que van desde 1 (totalmente en desacuerdo) a 7 (totalmente de acuerdo).

\section{Escala de Satisfacción Académica (Lent et} al., 2007). Se compone de siete ítems que se agrupan en un solo factor, el que se orienta a medir el bienestar y disfrute que perciben los estudiantes en su experiencia de aprendizaje (Lent et al., 2007). Se utilizó una versión adaptada al español que ha presentado niveles adecuados de validez y consistencia interna (Medrano, Fernández, \& Pérez, 2014). Los ítems se respondieron en una escala tipo Likert 7 puntos que van desde 1 (totalmente en desacuerdo) a 7 (totalmente de acuerdo). Para el análisis se consideraron las puntuaciones totales de la escala.

\section{Escala de Autoeficacia Académica} (Palenzuela, 1983). En su construcción y validación original mostró una estructura unidimensional y adecuadas propiedades psicométricas. En el proceso de validación de la escala realizada con una muestra de adolescentes de edades comprendidas entre 12 y 16 años de Educación Secundaria Obligatoria en España, confirmó una estructura unidimensional con un coeficiente de consistencia interna $\alpha=.89$ y fiabilidad test-retest de .87 (García-Fernández et al., 2010). La escala está compuesta por 10 ítems agrupados en un factor que miden las expectativas de autoeficacia en situaciones específicas del contexto educativo en estudiantes adolescentes. Los ítems son evaluados a través de una escala con formato tipo Likert de 4 puntos que van desde 1 (nunca) a 4 (siempre).

En su construcción y validación original, con una muestra de adolescentes de Educación Secundaria Obligatoria en España, confirmó una estructura unidimensional con un coeficiente de consistencia interna $\alpha=.89$ y fiabilidad test-retest de .87 (García-Fernández et al., 2010). Este instrumento fue validado en Chile por GarcíaFernández et al (2016) con estudiantes de educación secundaria $\left(1^{\circ}\right.$ a $4^{\circ}$ medio). Los resultados mostraron una buena consistencia interna de la escala $(\alpha=.88)$.

En esta investigación se utilizó la versión autorizada de este instrumento para su adaptación y aplicación con estudiantes universitarios. Los resultados del proceso de adaptación con estudiantes universitarios chilenos mostraron adecuados niveles de validez interna $\left(\chi^{2} / \mathrm{gl}=2.00\right.$; CFI $=.97 ; \quad$ IFI $=.97 ; \quad$ TLI $=.96 ; \quad$ RMSEA $=.058 ;$ RMR=.039), además, las puntuaciones presentaron niveles adecuados de fiabilidad $(\alpha=.87)$ (Del Valle, Díaz, Pérez, \& Vergara, 2018).

\section{Procedimiento}

La administración de las escalas se realizó en dependencias de la Universidad de Antofagasta, Chile. En primer lugar y antes de la aplicación de los instrumentos se contactó con jefes de carrera o directores de departamento para solicitar su colaboración y explicar los objetivos de la investigación, una vez obtenida su autorización se coordinó día y horario para la aplicación. Se solicitó la participación voluntaria y anónima de estudiantes entregándoles carta de consentimiento informado, documento que explicaba los objetivos del estudio, el tiempo de duración, indicaciones para responder los instrumentos y el resguardo de la confidencialidad tanto de los datos como de los resultados obtenidos. La aplicación se realizó de forma colectiva en la misma sala de clases. Se aplicó un consentimiento informado y firmado, determinando los riesgos y beneficios para los participantes en el estudio. La investigación fue autorizada por el Comité de Ética en Investigación Científica de la Universidad de Antofagasta.

\section{Análisis de los datos}

Para realizar el análisis descriptivo se calcularon la media, la desviación estándar y las puntuaciones $\mathrm{Z}$. La consistencia interna de los datos se evaluó a través del coeficiente alfa de Cronbach. Se consideraron valores $\alpha \geq 70$ para identificar una fiabilidad aceptable (Kaplan \& Saccuzzo, 2006). La correlación entre las variables se evaluó utilizando el coeficiente de correlación $r$ de Pearson.

La identificación de los perfiles motivacionales se realizó a través del análisis de perfil latente (LPA - Latent Profile Analysis). Esta técnica se basa en el ajuste de un modelo a los datos observados, donde los casos o personas se incluyen en el grupo más probable, de acuerdo a las respuestas obtenidas para cada variable observada. De esta manera, se considera una 
herramienta de análisis centrada en las "similitudes y diferencias entre las personas en lugar de las relaciones entre las variables" (Berlin, Williams, \& Parra, 2013, p.174).

Para determinar el número óptimo de grupos, se evaluó el ajuste de un conjunto de modelos de análisis de perfil latente, mediante la comparación iterativa de un modelo con $\mathrm{k}$ grupos con un modelo con k-1 grupos. La bondad de ajuste se evaluó a través de los siguientes índices y criterios (Ram \& Grimm, 2009): (a) criterio de Información de Akaike (AIC) y criterio de Información Bayesiano ajustado por el tamaño de la muestra (sBIC). El mejor modelo es el que presenta los valores más bajos de AIC y sBIC; (b) test de razón de verosimilitud de Lo-MendellRubin (LMRT) y test de razón de verosimilitud de bootstraping (BLRT). Estas pruebas comparan un modelo de $\mathrm{k}$ grupos con un modelo con $\mathrm{k}-1$ grupos. Un valor $\mathrm{p}<.05$ indica que el modelo con $\mathrm{k}$ grupos tiene un mejor ajuste que el modelo con k - 1 grupos; (c) entropía, utilizada para evaluar la calidad de la clasificación de las personas en los grupos. Esta medida varía de 0 a 1 , donde un valor más cercano a 1 indica una mejor clasificación; (d) tamaño de los grupos, el que se recomienda no ser inferior al $5 \%$ de la muestra.

A partir de los hallazgos del trabajo exploratorio realizado por Vansteenkiste et al. (2009), se identificó la correspondencia entre los perfiles motivacionales observados y los cuatro perfiles motivacionales propuestos desde la teoría de la autodeterminación (Deci \& Ryan, 2000). Por lo tanto, esta investigación se orientó al análisis confirmatorio de un modelo con las siguientes clases motivacionales latentes: (1) baja calidad, (2) baja cantidad, (3) alta cantidad, (4) alta calidad. Al igual que Vansteenkiste et al. (2009), se utilizaron la motivación autónoma y la motivación controlada como variables de agrupación. Además, se utilizó la prueba ANOVA unifactorial para evaluar diferencias significativas entre los perfiles motivacionales en cuanto a la satisfacción y la autoeficacia académica. Finalmente, se analizó el tamaño del efecto $(f)$ considerando los siguientes criterios: (1) efecto pequeño=.10; (2) efecto mediano=.25; y (3) efecto grande $=.40$ (Cárdenas \& Arancibia, 2014).

\section{Resultados}

\section{Análisis descriptivo y correlacional}

En la Tabla 1 se muestran los estadísticos descriptivos de cada una de las variables analizadas. Los resultados muestran que la motivación autónoma y la satisfacción académica presentaron los valores más altos y la motivación controlada presentó los valores más bajos.

Respecto de las correlaciones bivariadas, los resultados indicaron que la motivación autónoma tuvo una relación positiva y significativa con la satisfacción académica $(\mathrm{r}=.65, \quad p<.01)$ y la autoeficacia académica $(\mathrm{r}=.31, p<.01)$ Por otro lado, se observó que la motivación controlada tuvo una relación negativa y significativa con la motivación autónoma $(\mathrm{r}=-.25, \quad p<.01), \quad$ la satisfacción académica $(\mathrm{r}=-.32, \quad p<.01) \quad$ y la autoeficacia académica $(\mathrm{r}=-.21, p<.01)$ (ver Tabla $1)$.

Tabla 1. Estadísticos descriptivos y correlación entre variables

\begin{tabular}{|c|c|c|c|c|c|c|}
\hline Variables & $M$ & D.T. & 1 & 2 & 3 & 4 \\
\hline $\begin{array}{l}\text { 1. Motivación } \\
\text { autónoma }\end{array}$ & 5.82 & 1.02 & 1.00 & $-.25^{* *}$ & $.65^{* *}$ & $.31^{* *}$ \\
\hline $\begin{array}{l}\text { 2. Motivación } \\
\text { controlada }\end{array}$ & 2.12 & 1.13 & & 1.00 & $-.32^{* *}$ & $-.21^{* *}$ \\
\hline $\begin{array}{l}\text { 3. Satisfacción } \\
\text { académica }\end{array}$ & 5.77 & 0.87 & & & 1.00 & $.43^{* *}$ \\
\hline $\begin{array}{l}\text { 4. Autoeficacia } \\
\text { académica }\end{array}$ & 5.18 & 0.93 & & & & 1.00 \\
\hline
\end{tabular}

A partir de los resultados, se observó que mientras aumentó la autodeterminación de los estudiantes para el aprendizaje, también se incrementó la satisfacción y la autoeficacia académica. Por el contrario, en la medida que aumentó la motivación controlada para el aprendizaje, se observó la disminución de la satisfacción y la autoeficacia académica de los estudiantes.

\section{Análisis de perfil latente}

En la Tabla 2 se presentan los valores de los índices de ajuste para los modelos con 2, 3 y 4 clases. Los resultados apoyaron la solución de 4 perfiles motivacionales, ya que presentó los valores más bajos en AIC y sBIC. Además, los valores LRMT y BLRT fueron estadísticamente significativos $(p<.05)$ y el valor de entropía indica 
Tabla 2. İndices de ajuste

\begin{tabular}{lccccc}
\hline Clases & LRMT(p) & BLRT $(\mathrm{p})$ & AIC & sBIC & Entropía \\
\hline 2 & $136.70(.01)$ & $144.19(.01)$ & 2348.60 & 2354.95 & .90 \\
3 & $53.72(.06)$ & $56.67(.01)$ & 2297.94 & 2307.00 & .87 \\
4 & $76.76(.01)$ & $80.97(.01)$ & 2222.97 & 2234.75 & .89 \\
\hline
\end{tabular}

Fuente: Elaboración propia.

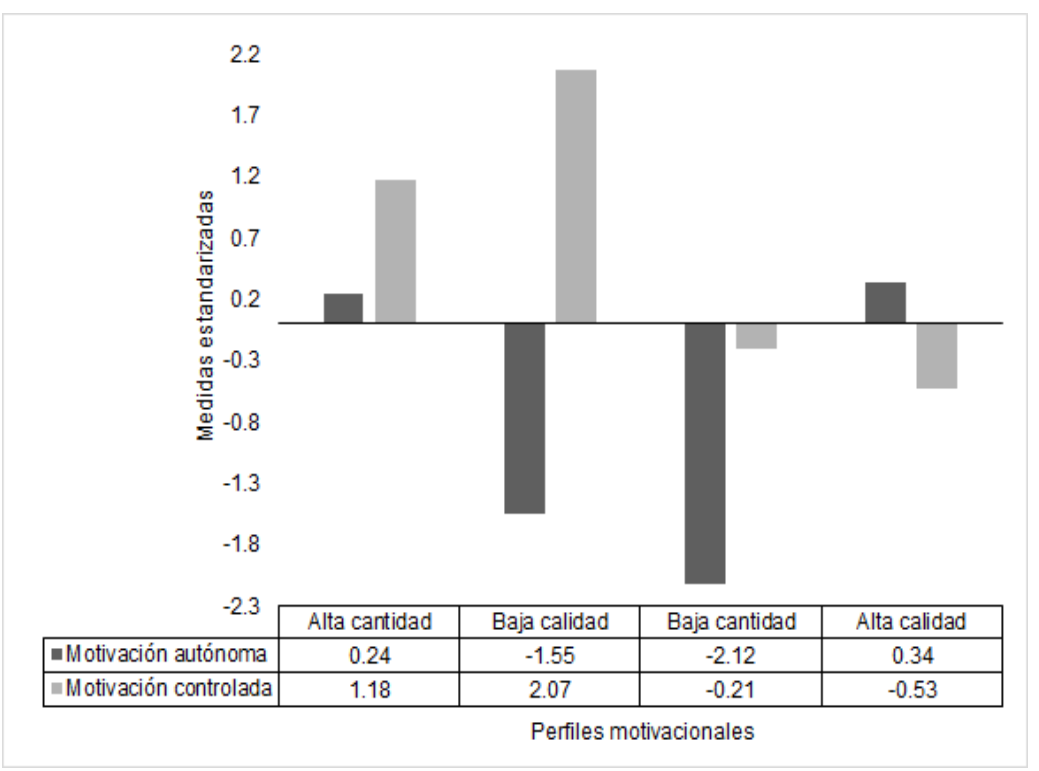

Figura 1. Perfiles motivacionales

Fuente: Elaboración propia.

Tabla 3. Puntuaciones medias y análisis de diferencias en función de los perfiles motivacionales

\begin{tabular}{|c|c|c|c|c|c|c|c|}
\hline \multirow[b]{2}{*}{ Variables } & \multicolumn{4}{|c|}{ Perfiles motivacionales } & \multirow[b]{2}{*}{$F$} & \multirow[b]{2}{*}{$f$} & \multirow[b]{2}{*}{$\mathrm{Eta}^{2}$ parcial } \\
\hline & $\begin{array}{c}\text { Baja } \\
\text { calidad } \\
1\end{array}$ & $\begin{array}{c}\text { Baja } \\
\text { cantidad } \\
2\end{array}$ & $\begin{array}{c}\text { Alta } \\
\text { cantidad } \\
3\end{array}$ & $\begin{array}{c}\text { Alta } \\
\text { calidad } \\
4\end{array}$ & & & \\
\hline $\begin{array}{l}\text { Satisfacción } \\
\text { académica }\end{array}$ & 4.31 & 4.99 & 5.20 & 5.28 & $F(3,433)=61.47$ & 0.75 & 0.30 \\
\hline $\begin{array}{l}\text { Autoeficacia } \\
\text { académica }\end{array}$ & 4.53 & 4.73 & 5.75 & 6.02 & $F(3,433)=10.60$ & 0.31 & 0.07 \\
\hline
\end{tabular}

Fuente: Elaboración propia. Nota. $f=$ Tamaño del efecto; ${ }^{*}$ diferencias significativas entre grupos $=4>1 ; 4>2 ; 4>3 ; 3>1 ; 3>2 ; 2<4 ; 2$ $<3 ; 1<4 ; 1<3 ;{ }^{* *}$ diferencias significativas entre grupos $=4>1 ; 3>1 ; 2>1 ; 1<4 ; 1<3 ; 1<2$

que el modelo tuvo un $89 \%$ de probabilidad de clasificar con precisión a las personas en las clases del modelo.

En la Figura 1 se muestran los perfiles motivacionales latentes identificados: (a) alta cantidad $(19.2 \%, \mathrm{n}=84)$, con puntuaciones altas en las variables motivacionales; (b) baja calidad (6.4\%, $\mathrm{n}=28$ ), con puntuaciones relativamente altas en motivación controlada y puntuaciones relativamente bajas en motivación autónoma; (c) baja cantidad (8.0\%, $\mathrm{n}=35)$, con puntuaciones bajas en las variables motivacionales; y (d) alta calidad (66.4\%, $\mathrm{n}=290$ ), con puntuaciones relativamente altas en motivación autónoma y puntuaciones relativamente bajas en motivación controlada (ver Figura 1). Finalmente, se observó un tamaño adecuado de los grupos, ya que fueron superiores al 5\% de la muestra $(n=22)$.

\section{Comparación de variables}

En la Tabla 3 se muestran los resultados del análisis de comparación entre los perfiles motivacionales en cuanto a la satisfacción y la autoeficacia académica. Los resultados indican diferencias estadísticamente significativas entre los perfiles motivacionales, observándose que el perfil con mayor grado de autodeterminación presentó los valores más altos de satisfacción y autoeficacia, mientras que el perfil motivacional con menor grado de autodeterminación presentó los valores más bajos (ver Tabla 2). 


\section{Discusión}

El objetivo de este estudio fue identificar los perfiles motivacionales de estudiantes universitarios, evaluando su relación con la satisfacción y la autoeficacia académica. Al igual que otras investigaciones, se observó que existe una relación positiva y directa entre la motivación autónoma y la satisfacción académica de los estudiantes universitarios, lo que estimula el desarrollo de comportamientos autodeterminados que facilitan la emergencia de distintos tipos de perfiles motivacionales latentes (Jeno \& Diseth, 2014; Méndez-Giménez et al., 2013). Además, los hallazgos muestran que cuando los estudiantes activan una motivación autónoma en el desarrollo de las actividades académicas, tienen una percepción positiva de sus creencias de autoeficacia. Esto concuerda con la perspectiva de la TAD, ya que la satisfacción de la necesidad de autonomía constituye un nutriente esencial para el crecimiento, integración y la salud psicológica de los individuos (Deci \& Ryan, 2000).

Por otro lado, los resultados del estudio permiten confirmar la emergencia de cuatro perfiles motivacionales relacionados con la calidad y cantidad de la motivación, tal como se propone desde la teoría de la autodeterminación (Sánchez-Oliva et al., 2015; Vansteenkiste et al., 2009; Vergara-Morales, Del Valle, Díaz, Matos, \& Pérez, 2019). De esta manera, si se considera que durante el desarrollo de las actividades académicas pueden coexistir diferentes tipos de motivación, las características motivacionales de los estudiantes se clasifican en torno a la calidad y cantidad de motivación. Es decir, los estudiantes activan un tipo de motivación académica, de acuerdo con la calidad y cantidad del interés, esfuerzo y persistencia que expresan en el proceso de enseñanza aprendizaje. A través de los perfiles latentes identificados, se infiere que mientras los estudiantes perciben una mayor autonomía e iniciativa personal para el desarrollo de las actividades académicas, se facilita la activación de una motivación autodeterminada hacia la meta de aprendizaje (Medellín, 2010).

Los hallazgos del estudio permiten concluir que los perfiles motivacionales latentes influyen en las creencias de autoeficacia. Considerando los resultados del trabajo realizado por Valle et al.,
(2015), es fundamental indagar el comportamiento de las creencias de autoeficacia de acuerdo a los perfiles motivacionales, ya que se observa una influencia sobre la percepción de las capacidades para organizar y ejecutar las acciones necesarias para realizar diferentes tipos de tareas (GuerreiroCasanova \& Polydoro, 2011). De esta manera, se infiere que el desarrollo de altos grados de disfrute y satisfacción por las actividades académicas incide en el desarrollo de una percepción positiva sobre las capacidades para lograr con éxito los resultados de aprendizaje. Esto permite indagar las implicancias cognitivas de la motivación en la educación universitaria.

Además, se concluye que los perfiles motivacionales latentes influyen en el grado de satisfacción académica que perciben los estudiantes, lo que podría afectar el logro de la meta de aprendizaje. Por lo tanto, es fundamental que los docentes se impliquen en los procesos motivacionales y académicos de los estudiantes, para facilitar el logro de los resultados académicos. Finalmente, se considera importante la implementación de programas de intervención que evalúen los perfiles motivacionales y las variables cognitivas que influyen en el proceso académico, para así contribuir al fortalecimiento de los aprendizajes.

\section{Referencias}

Alonso-Tapia, J., Panadero, E., \& Ruiz, M.A. (2014). Development and validity of the Emotion and Motivation Self-regulation Questionnaire (EMSR-Q): Intertwined effects between selfregulation styles and classroom motivational climate. Spanish Journal of Psychology, 17. doi:10.1017/sjp.2014.41

Álvarez, P., \& López, D. (2017). Estudios sobre deserción académica y medidas orientadoras de prevención en la universidad de la laguna (España). Paradigma, 38(1), 48-71.

Anaya-Durand, A., \& Anaya-Huertas, C. (2010). ¿Motivar para aprobar o para aprender? Estrategias de motivación del aprendizaje para los estudiantes. Tecnología, Ciencia, Educación, 25(1), 5-14.

Becerra-González, C. E., \& Reidl L. M. (2015). Motivación, autoeficacia, estilo atribucional y rendimiento escolar de estudiantes de 
bachillerato. Revista Electrónica de Investigación Educativa, 17(3), 79-93.

Berlin, K. S., Williams, N. A., \& Parra, G. R. (2013). An introduction to latent Variable Mixture Modeling (Part 1): Overview and cross-sectional latent class and latent profile analyses, Journal of Pediatric Psychology, 39(2), 174-187. doi:10.1093/jpepsy/jst084

Bernardo, A., González-Pienda, J. A., Cervero, A., Esteban, M., Navarro, J. I. \& Martín, C. (2018). Diversas formas de motivar en el proceso enseñanza-aprendizaje. Aprendizaje escolar desde la psicología (Coords. Navarro, J. I. y Martín, C.) Editorial: Pirámide.

Cárdenas, M., \& Arancibia, H. (2014). Potencia estadística y cálculo del tamaño del efecto en G*Power. Complementos a las pruebas de significación estadística y su aplicación en Psicología. Salud \& Sociedad, 5(2), 210-224. doi:10.22199/S07187475.2014.0002.00006

Cartagena, M. (2008). Relación entre la autoeficacia en el rendimiento escolar y los hábitos de estudio en el rendimiento académico en alumnos de Secundaria. REICE. Revista Iberoamericana sobre Calidad, Eficacia y Cambio en Educación, 6(3), 59-99.

Donoso, S., \& E. Schiefelbein, (2007) Análisis de los modelos explicativos de retención de estudiantes en la universidad: Una visión desde la desigualdad social. Estudios Pedagógicos de Valdivia, 33(1). doi:10.4067/s0718-07052007000100001

Deci, E. L., \& Ryan, R. M. (2008). Selfdetermination theory: A macrotheory of human motivation, development, and health. Canadian Psychology/ Psychologie canadienne, 49(3), 182-185. doi:10.1037/a0012801

Deci, E. L., \& Ryan, R. M. (2000). The "what" and "why" of goal pursuits: Human needs and the selfdetermination of behavior. Psychological Inquiry, 11(4), 227-268. doi:10.1207/S15327965PLI110401

Del Valle, M., Díaz, A., Pérez, M. V., \& Vergara, J. (2018). Análisis factorial confirmatorio Escala Autoeficacia Percibida en Situaciones Académicas (EAPESA) en universitarios chilenos. Revista Iberoamericana de Diagnóstico y Evaluación - e Avaliação Psicológica, 4(49), 97-106. doi:10.21865/ridep49.4.08

García-Fernández, J. M., Inglés, C. J., Vicent, M., Gonzálvez, C., Pérez Sánchez, A. M., \& Lagos San Martín, N. (2016). Validación de la Escala de Autoeficacia Percibida Específica de Situaciones Académicas en Chile y su relación con las estrategias de aprendizaje. Revista Iberoamericana de Diagnóstico y Evaluación - e Avaliação Psicológica, 41(1), 118-131.

García-Fernández, J. M., Ingés, C. J., Torregrosa, M. S., Ruiz-Estenan, C., Díaz-Herrero, A., Pérez-Fernández, E., \& MartínezMonteagudo, M. C. (2010). Propiedades psicométricas de la Escala de Autoeficacia Percibida Específica de Situaciones Académicas en una muestra de estudiantes españoles de Educación Secundaria Obligatoria. European Journal of Educational Psychology, 3(1), 61-74.

Guerreiro-Casanova, D., \& Polydoro, S., (2011). Autoeficácia na formação superior: Percepções durante o primeiro ano de graduação. Psicologia: Ciência e profissão, 31(1), 50-65.

doi:10.1590/s1414-98932011000100006

Gómez-López, M., Granero-Gallegos, A., BaenaExtremera, A., \& Abraldes, J. A. (2014). Análisis de los perfiles motivacionales y su relación con la importancia de la educación física en secundaria. Revista Iberoamericana de Diagnóstico y Evaluación - e Avaliação Psicológica, 2(38), 11-29.

Ram, N., \& Grimm, K. J. (2009). Growth mixture modeling: A method for identifying differences in longitudinal change among unobserved groups. International Journal of Behavioral Development, 33(6), 565-576. doi:10.1177/0165025409343765

Howard, J., Gagné, M., \& Bureau, J. (2017). Testing a continuum structure of selfdetermined motivation: A meta-analysis. Psychological Bulletin, 143(12), 1346-1377. doi:10.1037/bul0000125

Jeno, L. M., \& Diseth, ̊. (2014). A selfdetermination theory perspective on autonomy support, autonomous self-regulation, and perceived school performance. Reflecting Education, 9(1), 1-20. 
Kaplan, R., \& Saccuzzo, D. (2006). Pruebas psicológicas. Principios, aplicaciones y temas. México: Thomson.

Komarraju, M., \& Dial, C. (2014). Academic identity, self-efficacy, and self-esteem predict self-determined motivation and goals. Learning and Individual Differences, 32, 1-8. doi:10.1016/j.lindif.2014.02.004

Lent, R., Singley, D., Sheu, H., Schmidt, J., \& Schmidt, L. (2007). Relation of socialcognitive factors to academic satisfaction in engineering students. Journal of Career Assessment, 15(1), 87-97. doi: $10.1177 / 1069072706294518$

Litalien, D., Morin, A., Gagné, M., Vallerand, R., Losier, G., \& Ryan, R. (2017). Evidence of a continuum structure of academic selfdetermination: A two-study test using a bifactor-ESEM representation of academic motivation. Contemporary Educational Psychology, 51, 67-82. doi:10.1177/1069072706294518

Maroño, C. T., Deus, E. R., Mallo, S., \& Méndez, R. (2015). Percepción de autoeficacia, rendimiento académico y perfil vocacional en estudiantes de $4^{\circ}$ de ESO. Revista de Estudios e Investigación en Psicología y Educación, (03), 6. doi:10.17979/reipe.2015.0.03.139

Medellín, E., (2010). Contrastación de dos modelos motivacionales de autodeterminación para predecir la deserción en universitarios. Acta Colombiana de Psicología, 13(2), 57-68.

Medrano, L. A., Fernández, M., \& Pérez, E. (2014). Computerized Assessment System for Academic Satisfaction (ASAS) for first-year University Student. Electronic Journal of Research in Educational Psychology, 12(2), 541-562. doi:10.25115/ejrep.33.13131

Meece, J. L., Anderman, E. M., \& Anderman, L. H. (2006). Classroom goal structure, student motivation, and academic achievement. Annual Review of Psychology, 57(1), 487-503. doi:10.1146/annurev.psych.56.091103.070258 Méndez-Giménez, A., Fernández-Río, J., \& Cecchini-Estrada, J. A. (2012). Análisis de un modelo multiteórico de metas de logro, metas de amistad y autodeterminación en educación física. Estudios de Psicología, 33(3), 325-336. doi:10.1174/021093912803758110
Miñano, P., \& Castejón, J. L. (2011). Variables cognitivas y motivacionales en el rendimiento académico en Lengua y Matemáticas: Un modelo estructural. Journal of Psychodidactics, 16(2).

Moreno Murcia, J. A., \& Silveira Torregrosa, Y. (2015). Perfiles motivacionales de estudiantes universitarios. Procesos de estudio y satisfacción con la vida. Revista Electrónica Interuniversitaria De Formación Del Profesorado, 18(3), 169-181. doi:10.6018/reifop.18.3.200441

Moreno J., Silveira Y., \& Alias, A. (2015). Modelo predictivo para la mejora de la percepción de competencia y rendimiento académico en estudiantes universitarios. REDU: Revista de Docencia Universitaria, 13(2), 173. doi:10.4995/redu.2015.5443

Muñiz, J., Fidalgo, A. M., García-Cueto, E., Martínez, R., \& Moreno, R. (2005). Análisis de los ítems. Madrid, España: La Muralla, S.A

Oriol-Granado, X., Mendoza-Lira, M., Covarrubias-Apablaza, C.-G., \& MolinaLópez, V.-M. (2017). Positive emotions, autonomy support and academic performance of University students: The mediating role of academic engagement and self-efficacy. Revista de Psicodidáctica (English Ed.), 22(1), 45-53. doi:10.1387/revpsicodidact.14280

Palenzuela, D. (1983). Construcción y validación de una escala de autoeficacia percibida específica de situaciones académicas. Análisis y Modificación de conducta, 9(21), 185-219.

Pérez-Villalobos, M. V., Díaz-Mujica, A., González-Pienda, J. A., Núñez-Pérez, J. C., \& Rosário, P. (2009). Escala de metas de estudio para estudiantes universitarios. Interamerican Journal of Psychology, 43(3), 449-455.

Rosário, P. (2012). Predicción del rendimiento en matemáticas: Efecto de variables personales, socioeducativas y del contexto escolar. Psicothema, 24(2), 289-295.

Rothes, A., Lemos, M., \& Gonçalves, T. (2017). Motivacional profiles of adult learners. Adult Education Quarterly, 67(1), 3-29. doi:10.1177/0741713616669588

Ryan, R. M., \& Deci, E. L. (2000). Intrinsic and extrinsic motivations: Classic definitions and 
new directions. Contemporary Educational Psychology, 25(1), 54-67. doi:10.1006/ceps.1999.1020

Salmerón, H., Gutierrez, C., Salmeron, P., \& Rodríguez, S. (2011). Metas de logro, estrategias de regulación y rendimiento académico en diferentes estudios universitarios. Revista de Investigación Educativa, 29(2), 467-486.

Sánchez-Oliva, D., Leo, F., Amado, D., PulidoGonzález, J., \& García-Calvo, T. (2015). Análisis de los perfiles motivacionales y su relación con los comportamientos adaptativos en las clases de educación física. Revista Latinoamericana de Psicología, 47(3), 156166. doi:10.1016/j.rlp.2015.06.007

Servicio de Información de Educación Superior [SIES]. (2014). Panorama de la Educación Superior en Chile 2014. División de Educación Superior, Ministerio de Educación.

Silva, J. da, Beltrame, T. S., Viana, M. da S., Capistrano, R., \& Oliveira, A. do V. P. de. (2014). Autoeficácia e desempenho escolar de alunos do ensino fundamental. Psicologia Escolar e Educacional, 18(3), 411-420. doi:10.1590/2175-3539/2014/0183760

Stover, J., Uriel, F., De la Iglesia, G., Freiberg Hoffmann, A., \& Liporace Mercedes, F. (2014). Rendimiento académico, estrategias de aprendizaje y motivación en alumnos de Escuela Media de Buenos Aires. Perspectivas en Psicología: Revista de Psicología y Ciencias Afines, 11(2), 10-20.

Valenzuela, J., Muñoz Valenzuela, C., Silva-Peña, I., Gómez Nocetti, V., \& Precht Gandarillas, A. (2015). Motivación escolar: Claves para la formación motivacional de futuros docentes. Estudios Pedagógicos (Valdivia), 41(1), 351361. doi:10.4067/s0718-07052015000100021

Valle, A., Núñez, J. C., Cabanach, R. G., Rodríguez, S., González, J. A., \& Rosário, P. (2008). Capacidad predictiva de las metas académicas sobre el rendimiento en diferentes áreas curriculares. Revista Latinoamericana de Psicología, 40(1), 111-122.

Valle, A., Regueiro, B., Rodríguez, S., Piñeiro, I., Freire, C., Ferradás, M., \& Suárez, N. (2015). Perfiles motivacionales como combinación de expectativas de autoeficacia y metas académicas en estudiantes universitarios. European Journal of Education and Psychology, 8(1), 1-8. doi:10.1016/j.ejeps.2015.10.001

Vansteenkiste, M., Aelterman, N., De Muynck, G. J., Haerens, L., Patall, E., \& Reeve, J. (2018). Fostering personal meaning and selfrelevance: A self-determination theory perspective on internalization. The Journal of Experimental Education, 86(1), 30-49. doi:10.1080/00220973.2017.1381067

Vansteenkiste, M., Soenens, B., Sierens, E., Luyckx, K., \& Lens, W. (2009). Motivational profiles from a self-determination perspective: The quality of motivation matters. Journal of Educational Psychology, 101(3), 671-688. doi:10.1037/a0015083

Vansteenkiste, M., Sierens, E., Goossens, L., Soenens, B., Dochy, F., Mouratidis, A., Aelterman, N., Haerens, L., \& Beyers, W. (2012). Identifying conFigurations of perceived teacher autonomy support and structure: Associations with self-regulated learning, motivation and problem behavior Learning and Instruction, 22, 431-439. doi:10.1016/j.learninstruc.2012.04.002

Vergara-Morales, J. (2019). Influencia de factores motivacionales docentes en la satisfacción y desempeño académico de estudiantes universitarios (Tesis doctoral). Universidad de Concepción, Chile. doi:10.6018/analesps.35.3.320441

Vergara-Morales, J., Del Valle, M., Díaz, A., Matos, L., \& Pérez, M. V. (2019). Motivational profiles related to the academic satisfaction of university students. Anales De Psicología / Annals of Psychology, 35(3), 464-471. doi:10.6018/analesps.35.3.320441

Wormington, S. V., Corpus, J. H., \& Anderson, K. A. (2012). A person-centered investigation of academic motivationand its correlates in high school. Learning and Individual Differences, 22(4), 429-438. doi:10.13140/RG.2.2.22344.78088/1 